\title{
Zur Änderung der türkischen Verfassung durch das Gesetz Nr. 4709 vom 3. Oktober 2001
}

\author{
Anmerkungen aus der Sicht einer Strafrechtlerin ${ }^{1}$
}

Von Silvia Tellenbach, Freiburg i.Br.

Dem Inkrafttreten der türkischen Verfassung von 1982 waren schwierige Jahre vorausgegangen, die deutliche Spuren in dem neuen Gesetzeswerk hinterließen. Seit Mitte der Siebziger Jahre hatten gewalttätige Auseinandersetzungen zwischen links- und rechtsradikalen Gruppen ständig zugenommen, 1980 rechnete man mit täglich 20 Toten durch Terroranschläge. Staatliches Handeln war weitgehend gelähmt; daß es im Frühjahr in über 100 Wahlgängen nicht gelang, nach Ende der Amtszeit des alten einen neuen Staatspräsidenten zu wählen, ist nur ein Symptom dafür. Am 12. September 1980 griff das Militär ein. Einerseits ging eine Verhaftungswelle durch das Land. Parteien, Vereine, Gewerkschaften wurden zunächst geschlossen. Die gesetzgeberischen Kompetenzen wurden vom Nationalen Sicherheitsrat wahrgenommen. Auf der anderen Seite war die große Masse der Bevölkerung - was heute oft vergessen wird - erleichtert, daß sie wieder sicher auf die Straße gehen konnte.

Das türkische Militär verstand sich seit langem als der Garant von Fortschritt und Verwestlichung. Seit dem 19. Jahrhundert war das Militär die Institution, durch die nicht nur militärtechnische Neuerungen aus Europa ins osmanische Reich eingedrungen waren, sondern auch viele kulturelle Errungenschaften bis hin zu westlicher Musik und Malerei. Auch Atatürk kam bezeichnenderweise aus dem Militär. Das türkische Militär sah sich daher auch als der Bewahrer von Demokratie und fuhr stets dann - mit harter Hand - dazwischen, wenn das staatliche Leben - Regierung und Parlament - nicht mehr funktionierte. Es sah seine Aufgabe nicht darin, lange Militärregime zu errichten, sondern so rasch wie möglich einer zivilen Regierung wieder auf die Füße zu helfen, wenn auch nicht in der Art, die wir uns vorstellen würden.

Auch nach der Intervention von 1980 wurde bereits nach einem Jahr eine Verfassunggebende Versammlung einberufen, die aus dem Nationalen Sicherheitsrat und einer beratenden Versammlung von 120 Mitgliedern bestand. Sie arbeitete die Verfassung aus, die 
dann 1982 in Kraft trat. Im Herbst 1983 fanden Parlamentswahlen statt, am 6. Dezember 1983 trat das Parlament zusammen. Das markierte das Ende der Militärregierung.

Die neue Verfassung, ein detailreiches Werk mit einer Präambel, 177 teilweise sehr umfangreichen Artikeln und 16 Übergangsartikeln, entsprach zwar in den äußeren Strukturen mit Grundrechtsteil, Staatsorganisationsteil etc. dem Muster europäischer Verfassungen, trug inhaltlich aber Züge, die in hohem Maße autoritär waren und sich mit einer modernen freiheitlich-demokratischen Grundordnung nicht vertrugen. Auch gegenüber der sehr liberalen Verfassung von 1961 bedeuteten sie einen deutlichen Rückschritt. So wurden z.B. Grundrechte und Grundfreiheiten zwar grundsätzlich garantiert, die Möglichkeiten ihrer Beschränkung aber weit gefasst (Art. 12, 13). Praktisch konnte aus Gründen eines staatlichen Interesses jedes Grundrecht beschränkt werden, auch wenn als Schrankenschranken die Bedingung vorgesehen war, daß Beschränkungen im Einklang mit Wort und Geist der Verfassung und den Erfordernissen einer demokratischen Gesellschaft stehen müßten. Ebenso wurde das Verbot des Mißbrauchs von Grundrechten aufgestellt und das Verbot, Verfassungsvorschriften so auszulegen, als ob sie das Recht zur Beseitigung der Grundrechte anderer gäben. Hier findet man zwar in der türkischen Lehre häufig Hinweise auf Artikel 18 GG. ${ }^{2}$ Der entscheidende Unterschied ist aber, daß nach dem Grundgesetz die Verwirkung eines Grundrechts nach einem genau geregelten Verfahren ausschließlich vom Verfassungsgericht ausgesprochen werden kann, während die türkische Vorschrift nur auf den Erlaß von Strafvorschriften verweist und alles andere dem einfachen Gesetzgeber überläßt. Das hatte zur Folge, daß in der Diskussion um das Bestehen von Strafvorschriften im Staatsschutzbereich z.B. bei Propagandadelikten immer wieder mit einem diesbezüglichen Verfassungsauftrag durch Art. 14 II argumentiert wurde. - Auch z.B. die Vorschriften zum Parteienrecht (Art. 68 f.) brachten Einschränkungen und die Vorschriften zum Vereins- oder Demonstrationsrecht (Art. 33, 34) waren so gefaßt, daß sie Einschränkungen durch einfachgesetzliche Regelungen ermöglichten oder zumindest nicht verhinderten. Der viel zitierte, auch nach Ende der Militärregierung weiterbestehende Nationale Sicherheitsrat, zwar unter dem Vorsitz des Staatspräsidenten, aber mit einem starken Gewicht der obersten Militärführung, galt weiterhin als das eigentlich maßgebliche Zentrum der Macht. Schließlich verbot ein Übergangsartikel die Überprüfung der Verfassungsmäßigkeit von Gesetzen, die in der Zeit seit dem Militärputsch bis zum Zusammentreten des neuen Parlaments in Kraft gesetzt worden waren, also für die drei Jahre vom 12. September 1980 bis zum 6. Dezember 1983. Das betraf so heikle Gesetze wie das Parteiengesetz, das Vereinsgesetz, das Demonstrationsgesetz, das Gesetz über Gewerkschaften und Arbeitgeberverbände, das Gesetz über Tarifvertrag und Streik oder das Gesetz, das in Deutschland unter dem vergröbernden Namen Sprachenverbotsgesetz bekannt geworden ist. 
Seit mehreren Jahren ist die Aufnahme der Türkei in die EU ein Thema auf der türkischen Tagesordnung. Wie bekannt gibt es einen Kriterienkatalog ("Kopenhagener Kriterien”), den die Türkei erfüllen muß, wenn sie den Schritt nach Europa vollziehen will. Seit Jahren gibt es, meist hinter den Kulissen, auch einen erheblichen Druck auf die Türkei, ihre Gesetze den europäischen Standards anzupassen, wo sie diesem noch nicht entsprechen und vor allem - noch wichtiger, aber auch weitaus schwieriger - die gesetzlichen Regelungen in die Rechtswirklichkeit umzusetzen. Aus diesem Grund hat es schon seit Jahren immer wieder Gesetzesänderungen mit diesem Hintergrund gegeben, insbesondere im Bereich des Strafprozeßrechts, aber auch in anderen Gesetzen. Am 19. März 2001 hat die türkische Regierung als Reaktion auf die Beitrittspartnerschaft der EU-Kommission ein Nationales Programm aufgestellt, ${ }^{3}$ das die Maßnahmen aufführt, die kurzfristig, d.h. binnen eines Jahres, und mittelfristig zur Erfüllung der Kriterien für eine EU-Mitgliedschaft zu treffen sein würden. Auch eine umfangreiche Verfassungsänderung erwies sich dabei als unausweichlich. Nach langer Diskussion erging schließlich am 3. Oktober 2001 die Änderung von 34 Artikeln der Verfassung, bei der, wenn man die - meist magere - Gesetzesbegründung liest, bei sehr vielen Artikeln eine allgemeine Bezugnahme auf die Europäische Menschenrechtskonvention, bei einer Reihe von Vorschriften auch sogar auf einzelne Artikel der Konvention zu finden ist.

Im folgenden sollen hier die Änderungen im Mittelpunkt stehen, die besonders auf das Straf- und Strafprozeßrecht ausstrahlen; weitere, durchaus nicht alle Änderungen können nur kurz angerissen werden.

An der Spitze der Verfassung steht eine ausführliche Präambel, welche die kemalistische Staatsideologie in einer überhöhten Art zum Ausdruck bringt. Laut Art. 176 ist sie als integraler Bestandteil des Verfassungstextes zu verstehen und daher auch zur Auslegung anderer Verfassungsvorschriften heranzuziehen. ${ }^{4}$ In dieser Präambel fand sich folgender Passus: "in dem Gedanken, dem Glauben und der Entschlossenheit, (...), dass keine Meinung und Ansicht gegenüber den türkischen nationalen Interessen, der türkischen Existenz, dem Grundsatz der Unteilbarkeit von Staatsgebiet und Staatsvolk, den geschichtlichen und ideellen Werten des Türkentums und dem Nationalismus, den Prinzipien und Reformen sowie dem Zivilisationismus Atatürks geschützt wird und heilige religiöse Gefühle, wie es das Prinzip des Laizismus erfordert, auf keine Weise mit den Angelegenheiten und der

Amtsblatt v. 24.3.2001 Nummer 24352 a.

4 Rumpf, Christian, Das türkische Verfassungssystem - Eine Einführung mit vollständigem Verfassungstext, Wiesbaden, 1996, S. 99. Zitate aus der Verfassung von 1982 werden im Folgenden nach dieser Übersetzung zitiert, Zitate der Neufassung seit der Änderung vom 3. Oktober 2001 nach Rumpf, Christian, Verfassung der Republik Türkei (Übersetzung), http://www.tuerkeirecht.de/Verfassung2001.pdf. 
Politik des Staates vermischt werden (...)",5. Aus diesem Passus ließ sich die grundlegende verfassungsmäßige Akzeptanz von Meinungsäußerungsdelikten ablesen, und in einer politischen Lage, in der eine Erweiterung der Meinungsäußerungsfreiheit als zwingendes Gebot für einen EU-Beitritt auf der Tagesordnung stand, drängte sich die Frage einer Änderung auf. Der Entwurf der Verfassungsänderung sah vor, die Begriffe "Meinung und Ansicht" durch den Begriff Aktion (eylem) zu ersetzen. Damit sollte deutlich gemacht werden, dass eine Meinungsäußerung allein sehr wohl geschützt sein, sondern nur noch ein aktives Tun aus dem Schutzbereich heraus- fallen sollte. Dieser Begriff wurde jedoch bereits im Vorfeld der Parlamentsabstimmung in der Verfassungskommission durch das Wort "Tätigkeit" (faaliyet) ersetzt, das zwar ebenfalls aktives Handeln bezeichnet, Meinungsäußerungen aber nicht unbedingt ausschließt.

Zwei Artikel wurden geändert, die eine Schlüsselstellung für die Wahrnehmung von Grundrechten und -freiheiten durch die Bürger einnehmen, Art. 13 und Art. 14.

Grundrechte und Grundfreiheiten sind in Art. 12 garantiert. Sie alle können unter bestimmten Umständen beschränkt werden. Diese Beschränkungsgründe wurden in der alten Fassung des Art. 13 folgendermaßen aufgezählt: "Schutz der unteilbaren Einheit von Staatsgebiet und Staatsvolk, der nationalen Souveränität, der Republik, der nationalen Sicherheit, der öffentlichen Ordnung, der Sicherheit der Allgemeinheit, des öffentlichen Interesses, des Sittengesetzes und der öffentlichen Gesundheit und aus besonderen Gründen, welche darüber in den entsprechenden Artikeln der Verfassung vorgesehen sind, (...)" (Art. 13 I) Bei diesem Katalog bot vor allem der Grund des öffentlichen Interesses ein weites Feld für Beschränkungen. Andererseits enthielt auch Art. 13 a.F. gewisse Schrankenschranken: Einschränkungen mußten im Einklang mit Wortlaut und Geist der Verfassung stehen, sie durften den Erfordernissen einer demokratischen Gesellschaftsordnung nicht entgegenstehen, nicht außerhalb ihres bestimmungsgemäßen Zwecks gebraucht werden und mußten durch Gesetz erfolgen.

Die Neufassung des Art. 13 läßt den Katalog von Einschränkungen aus allgemeinen Gründen entfallen, erlaubt sie nur noch aus den Gründen, die in den betreffenden Verfassungsbestimmungen angeführt sind und wie bisher schon nur durch Gesetz. Als Schrankenschranken finden sich weiterhin das Gebot des Einklangs mit Wortlaut und Geist der Verfassung sowie das Verbot, den Erfordernissen einer demokratischen Gesellschaftsordnung zu widersprechen. Neu ist dagegen die ausdrückliche Erwähnung des Verbots, den Erfordernissen einer laizistischen Republik zu widersprechen. Zwei weitere Schrankenschranken werden jetzt jedoch in die Bestimmung des Art. 13 aufgenommen. Zunächst eine Wesensgehaltsgarantie für die Grundrechte und Grundfreiheiten. Eine solche hatte es bereits in Art. 11 der Verfassung von 1961 gegeben, sie war dann aber 1982 durch die Erfordernisse einer

5

Rumpf (Fn. 4), S. 317. 
demokratischen Gesellschaftsordnung ersetzt worden, mit der Begründung, daß dieses Merkmal eindeutiger und damit leichter anzuwenden sei. ${ }^{6}$ Freilich stellte sich auch hier die Frage, was unter einer demokratischen Gesellschaftsordnung zu verstehen sei, eine Verfassungsordnung wie in der Verfassung von 1982 festgeschrieben oder die allgemeinen und universellen Grundsätze einer modernen Demokratie. Die Rechtsprechung des Verfassungsgerichts war nach anfänglicher Bevorzugung der ersten Meinung bald zur Bevorzugung der zweiten Auffassung gelangt und hatte sogar das Merkmal der Erfordernisse einer demokratischen Gesellschaftsordnung zunehmend mit einer Wesensgehaltsgarantie gleichgesetzt. ${ }^{7}$ So war zumindest der Boden für die jetzt erfolgte Aufnahme in Art. 13 schon vorbereitet. Ähnliches gilt für die zweite, neu in Art. 13 vorgesehene Schrankenschranke, den Verhältnismäßigkeitsgrundsatz. Er hatte sich zwar bereits an anderer Stelle in der Verfassung gefunden, nämlich in Art. 15, der Einschränkungen in Kriegs-, Mobilmachungs-, Ausnahmezustands- und Notstandszeiten regelt, nicht aber in Art. 13 und auch nicht in Art. 11 der Verfassung von 1961. Jedoch hatte auch hier das Verfassungsgericht ihn bereits in zahlreichen Entscheidungen angewendet und damit Vorarbeit auch für die Verankerung in Art. 13 geleistet. ${ }^{8}$

Art. 14, der den Mißbrauch von Grundrechten und -freiheiten verbietet, wird neu gefaßt und gestrafft. Er verbietet nunmehr Aktivitäten zur Zerstörung der unteilbaren Einheit von Staatsgebiet und Staatsvolk und der auf die Menschenrechte gegründeten demokratischen und laizistischen Republik. Während in der früheren Fassung der Gebrauch von Grundrechten zu bestimmten Zwecken schlechthin verboten und die dafür notwendige Form des Mißbrauchs nicht näher beschrieben war, wird auch hier - wie in der Präambel - der Begriff Aktivitäten verwendet, ein vorsichtiger Schritt, um reine Meinungsäußerungen zumindest zurücktreten zu lassen. Der detaillierte Katalog der verbotenen Zwecke wird also durch eine neue generalklauselartige Formulierung ersetzt, ohne daß man sagen kann, daß bestimmte Werte, die in der alten Fassung geschützt waren, in der neuen nicht mehr geschützt seien. ${ }^{9}$

Deutlicher als bisher wird ausgedrückt, daß nicht nur der einzelne Bürger, sondern auch der Staat keine Vorschrift der Verfassung so auslegen darf, daß ein Entzug oder eine Beschränkung die Folge ist, die über das verfassungsmäßig vorgesehene Maß hinaus geht (Art. 14). Auch hier ist eine der Stellen, an denen der Einfluß der EMRK faßbar wird, hier des Art. 17.

Özbudun (Fn. 6), S. 107. 
Die vorsichtige Lockerung der Einschränkungen in Art. 13, 14 führte im Laufe des vergangenen Jahres zur Änderung einiger Straftatbestände, welche Meinungsäußerungen betrafen. Bei Art. 7 II Antiterrorgesetz, der die Propaganda für eine terroristische Vereinigung unter Strafe stellt, wurde das Merkmal eingefügt, daß dabei zum Einsatz terroristischer Methoden aufgestachelt werden müsse. ${ }^{10}$ Bei Art. 8 Antiterrorgesetz, der separatistischen Propaganda, wurde dagegen die Aufstachelung zum Einsatz terroristischer Methoden nur als Strafschärfungsgrund eingefügt, ${ }^{11}$ der Grundtatbestand der separatistischen Propaganda verlangt also nach wie vor keine Propaganda für Gewalt. Art. 312 II StGB, das öffentliche Aufhetzen der Bevölkerung zu $\mathrm{Haß}$ und Feindschaft gegeneinander aus Gründen der Klasse, Rasse, Religion, Konfession oder Region, dessen Anwendung besonders gegenüber Islamisten und PKK-Sympathisanten in den letzten Jahren sehr zugenommen hat, wurde im Tatbestand begrenzt auf Fälle, bei denen die Tat zur Gefährdung der öffentlichen Ordnung geeignet ist. ${ }^{12}$ Wieweit das zu einem Rückgang der Verfahren führt, bleibt abzuwarten. Bei Art. 159, der Verunglimpfung bestimmter staatlicher Institutionen und Werte, wurde die Höchststrafe von 6 Jahren Zuchthaus auf drei Jahre Gefängnis herabgesetzt, eine Änderung, die wenig praktische Bedeutung hat, weil in der Türkei im Regelfall sowieso die Mindeststrafe verhängt wird, die hier bei einem Jahr liegt. ${ }^{13}$ Auch eine in deutschen Zeitungen viel beachtete Ergänzung des Tatbestands bringt letztlich nichts Neues: Meinungsäußerungen, die ohne die Absicht der Verunglimpfung der in Art. 159 genannten Institutionen und Werte, sondern nur mit der Absicht der Kritik erfolgen, erfüllen den Tatbestand nicht. ${ }^{14}$ Das entspricht aber seit langem der höchstrichterlichen Rechtsprechung zu Art. 159 und dem gesamten System der Beleidigungsdelikte. ${ }^{15}$

Im Pressegesetz (Gesetz Nr. 5680 v. 15.7.1950) wurden zahlreiche mit Freiheitsstrafen bedrohte Verstöße auf Geldstrafen reduziert. Dabei handelt es sich jedoch vorwiegend um Presseordnungsdelikte, nur in wenigen Fällen um Presseinhaltsdelikte. - Ganz gestrichen wurde Art. 31. ${ }^{16}$ Dieser sah vor, daß der Ministerrat die Einfuhr und Verteilung von Schriften in der Türkei verbieten konnte, die im Ausland erscheinen waren und gegen die unteilbare Einheit von Staatsgebiet und Staatsvolk, die nationale Souveränität, die Existenz der Republik, die nationale Sicherheit, die öffentliche Ordnung, die allgemeine Ruhe, das öffentliche Interesse, die allgemeinen Sitten und die öffentliche Gesundheit gerichtet waren

Art. 3 Gesetz Nr. 4744 v. 6.2.2002.

Art. 4 Gesetz Nr. 4744 v. 6.2.2002.

Art. 2 Gesetz Nr. 4744 v. 6.2.2002.

Art. 1 Gesetz Nr. 4744 v. 6.2.2002.

Art. 2 Gesetz Nr. 4771 v. 3.8.2002.

$\mathrm{Zu}$ den strafrechtlichen Änderungen vgl. demnächst eingehender Tellenbach, Silvia, Einführung ins türkische Strafrecht, im Erscheinen.

16

Art. 9, 12 Gesetz Nr.4777 v.3.8.2002. 
(I). Wer derartige Schriften trotz eines solchen Verbots wissentlich in die Türkei einführte, sie dort vertrieb, abdruckte oder übersetzte, war mit drei Monaten bis zu einem Jahr Gefängnis und Geldstrafe zu bestrafen (Art. 31 III).

Die bedeutsamste Änderung im Strafrecht betraf die Todesstrafe. Ihre Abschaffung war schon lange eines der heißesten Themen in der öffentlichen Diskussion in der Türkei. De facto war sie zwar seit 1984 nicht mehr vollstreckt worden, aber als Möglichkeit stand sie eben nach wie vor im Gesetz und hinderte damit auch weiterhin die Auslieferung von Straftätern an die Türkei, wenn die Gefahr der Todesstrafe drohen könnte. Letztlich schieden sich die Geister am Fall Öcalan. Die rechte Partei MHP war nicht bereit, auf eine Hinrichtung Öcalans zu verzichten. Sie wäre zu einer Abschaffung der Todesstrafe nur nach einer Vollstreckung der Todesstrafe gegen Öcalan bereit gewesen. Hier waren die Fronten so erstarrt, daß bei der Verfassungsänderung eine völlige Abschaffung nicht zu erreichen war. Die Formel, auf die man sich schließlich einigte, war, daß die Todesstrafe zwar unzulässig sein sollte, jedoch außer in Fällen des Krieges, unmittelbar drohender Kriegsgefahr oder terroristischer Straftaten. Das Erhaltenbleiben der Todesstrafe bei terroristischen Straftaten war jedoch mit dem 6. Zusatzprotokoll zur EMRK nicht vereinbar, das die Todesstrafe nur noch bei Krieg und drohender Kriegsgefahr hinnahm. Dieses hatte die Türkei allerdings nie unterschrieben. Wenn man das türkische Strafgesetzbuch durchsah, so konnte man feststellen, daß die dort vorgesehenen Todesstrafen fast alle bereits auf den Fall des Krieges, der Kriegsgefahr oder des Terrorismus beschränkt sind. Die einzige Ausnahme war Mord (Art. 450 StGB). ${ }^{17}$ Eine Strafgesetzänderung war also erforderlich; jedoch konnten die Gerichte zunächst durch die Anwendung des unbenannten Strafmilderungsgrundes des Art. 59 II StGB zum Erlaß von Urteilen kommen, die der neuen verfassungsrechtlichen Lage entsprachen.

Nachdem Anfang August 2002 die Abgeordneten aus dem Urlaub zurückgerufen worden waren, um zu beschließen, daß am 3. November vorzeitig Neuwahlen stattfinden würden, war die Sorge verbreitet, daß bei der vorhersehbaren Einschränkung der Handlungsfähigkeit von Parlament und Regierung kurz vor und nach den Wahlen die Zeit nicht mehr ausreichen würde, um rechtzeitig vor dem nächsten Fortschrittsbericht der EU-Kommission zum Erlaß der unausweichlichen Reformgesetze zu kommen. In dieser Situation unternahm die Mutterlandspartei (ANAP) einen weiteren Vorstoß, bei dem es noch wenige Tage nach dem Beschluß über die Neuwahlen in einer Marathonsitzung gegen den erbitterten Widerstand der nationalistischen MHP gelang, das lang diskutierte Reformpaket zu verabschieden. Durch dieses wurde die Todesstrafe in den einfachen Gesetzen außer in Zeiten von Krieg und Kriegsgefahr abgeschafft und zwar auch für terroristische Taten. ${ }^{18}$ Diese Regenennen. 
lung ging also weiter als die ein Dreivierteljahr vorher verabschiedeten verfassungsrechtlichen Vorgaben. Somit steht die türkische Rechtslage jetzt im Einklang mit dem 6. Zusatzprotokoll zur EMRK. ${ }^{19}$ Damit wurde das Leben von Öcalan und einer Reihe von Inhaftierten, die zum Tode verurteilt waren, endgültig gerettet. Daß diese Änderung weiter ging als die Verfassungsänderung vom 3. Oktober 2001, ist vor allem darauf zurückzuführen, daß dafür die einfache Mehrheit der anwesenden Parlamentsmitglieder ausreichte (Art. 96 I), während eine Verfassungsänderung einer Mehrheit von drei Fünfteln der Gesamtheit aller Mitglieder des Parlaments bedarf (Art. 175). Nach den Mehrheitsverhältnissen im türkischen Parlament war die Dreifünftelmehrheit für eine Verfassungsänderung nicht zu erreichen, weil die nationalistische MHP der Abschaffung der Todesstrafe, wie bereits erwähnt, nur nach vorheriger Hinrichtung Öcalans zugestimmt hätte. Der Terrorismusvorbehalt war also gewissermaßen der Preis, der dafür zu zahlen war, daß die Todesstrafe überhaupt in der Verfassung eingeschränkt werden konnte. ${ }^{20}$ In der Folgezeit wurde jedoch, besonders auch aus der Rechtswissenschaft, darauf hingewiesen, daß eine Abschaffung der Todesstrafe durchaus auch im Wege eines einfachen Gesetzes erfolgen könne. Die Regelung in der Verfassung würde dem Gesetzgeber nur die Erlaubnis geben, unter bestimmten Umständen die Todesstrafe vorzusehen, verpflichte ihn aber nicht dazu, sondern überließe es vielmehr seinem Ermessen, ob er von dieser Möglichkeit Gebrauch mache. ${ }^{21}$ Diese Lösung, die dann ja auch gewählt wurde, hatte eben den Vorteil, daß ein entsprechendes Gesetz ohne Zustimmung der MHP die nötigen Mehrheiten fand.

Die Verfassung sah bisher ein Amnestieverbot für Personen vor, die "wegen Taten gem. Art. 14 der Verfassung verurteilt waren (Art. 87)". Da aber Art. 14 selbst keine Straftatbestände enthielt, war es schon längst ein Kritikpunkt, daß zwar offensichtlich war, daß Amnestien von Staatsschutzdelikten nicht erfolgen durften, daß aber nicht deutlich war, welche Delikte nun genau darunter fielen. ${ }^{22}$ Die Aufnahme dieser Vorschrift in die Verfassung läßt sich historisch erklären. 1974 kamen im Laufe einer Amnestie zahlreiche Häftlinge frei, die nach der Militärintervention von $1971 \mathrm{zu}$ langjährigen Freiheitsstrafen verurteilt worden waren. Das wurde in vielen Kreisen dafür verantwortlich gemacht, daß bald darauf terroristische Straftaten in der Türkei erheblich zunahmen. Dergleichen wollte man für die Zukunft ausschließen. - In der Praxis führte das Verbot jedoch dazu, daß Amnestien, wenn sie trotzdem zumindest geringfügige Staatsschutzdelikte miterfassen

Mittlerweile wurde jedoch das 13. Zusatzprotokoll zur EMRK verabschiedet, das die Todesstrafe auch bei Krieg und unmittelbarer Kriegsgefahr für unzulässig erklärt, und es ergehen bereits die ersten Mahnungen an die Türkei, sie solle ihre Rechtslage an das 13. Zusatzprotokoll anpassen. Ein listiges Offenhalten eines Hinterpförtchens für die Todesstrafe in Terrorismusfällen, wie verschiedentlich in der Presse gemutmaßt, ist darin nicht zu sehen.

21 So der Istanbuler Strafrechtslehrer Dönmezer, http://www.nethaber.com (20.6.2002).

22 Vgl. Özbudun (Fn. 6), S. 297. 
wollten, ${ }^{23}$ nicht in der üblichen Form von Amnestien erfolgten, sondern auf eine komplizierte strafvollzugsrechtliche Lösung auswichen und den Weg einer sehr frühzeitigen Entlassung auf Bewährung wählten. ${ }^{24}$ Die Änderung der Verfassung vom vergangenen Oktober ließ jetzt dieses Amnestieverbot entfallen, schrieb jedoch in einer Übergangsvorschrift vor, daß das nur für Delikte nach Inkrafttreten der Verfassungsänderung gelten sollte. Künftige Amnestien in der Türkei werden also die gewohnten Formen von Amnestiegesetzen haben können. Allerdings wurde für Amnestien eine qualifizierte Mehrheit von drei Fünfteln der Gesamtzahl der Abgeordneten eingeführt.

Ein erheblicher Teil der Verfassungsänderungen bezieht sich auf Regelungen, die für den Strafprozeß wichtig sind. Dabei ist das Interessante, daß in vielen Fällen bereits die strafprozessuale Regelung der Verfassung vorausgegangen ist und die Verfassung jetzt gewissermaßen festschreibt, was das einfache Gesetz schon enthält.

In Art. 36 wird mit dem Recht auf Verteidigung auch die Garantie eines fairen Verfahrens aufgenommen, laut der Gesetzesbegründung, um damit den Bestimmungen internationaler Abkommen Rechnung zu tragen, denen die Türkei beigetreten ist und die ein faires Verfahren garantieren. Hierbei ist vor allem Art. 6 EMRK gemeint. Festzuhalten bleibt jedoch, dass eine Reihe der einzelnen Anforderungen des Art. 6 EMRK bereits in der türkischen Verfassung verankert ist, so z.B. die richterliche Unabhängigkeit (Art. 138 I), die Öffentlichkeit der Hauptverhandlung (Art. 141) oder die Unschuldsvermutung (Art. 38 IV). Das faire Verfahren, insbesondere die Unabhängigkeit der Gerichte, gehört aber derzeit zu den Fragen, die in der Wissenschaft, aber auch in der Öffentlichkeit immer wieder diskutiert werden. Das hat es gewiß begünstigt, dass das Prinzip des fairen Verfahrens in die Verfassung aufgenommen wurde. - Art. 17 III enthält von jeher das Verbot von Folter und Mißhandlung. Die Folter ist auch der düstere Hintergrund des neuen Art. 38 VIII: Die Unzulässigkeit der Verwendung von rechtswidrig erlangten Beweismitteln ist bereits seit 1992 in Art. 254 II StPO vorgesehen; jetzt erhält sie Verfassungsrang. Durch das Verbot der Verwendung von erfolterten Beweismitteln soll ein möglicher Anreiz für Folter genommen werden. Von größerer Bedeutung für die Verhinderung von Folter könnte aber eine Vorschrift werden, die kürzlich ins Beamtengesetz eingefügt worden ist (Art. 13 II): Wird der türkische Staat vom Europäischen Gerichtshof für Menschenrechte zu Schadenersatzleistungen wegen Folter oder unmenschlicher und erniedrigender Behandlung verurteilt, so hat der Staat ein Rückgriffsrecht gegen den verantwortlichen Beamten. ${ }^{25}$

So umfaßte die letzte türkische Amnestie durch das Gesetz Nr. 4616 v. 21.12.2000 die Unterstützung einer bewaffneten Bande (Art. 169 StGB) sowie die Straftaten nach dem Antiterrorgesetz Nr. 3713 v. 12. 4. 1991, also z.B. die separatistische Propaganda des Art. 8. 
Bei festgenommenen oder verhafteten Personen sah die Verfassung vor, daß eine Person nach spätestens 48 Stunden zuzüglich der Wegezeit, um den nächsten Richter zu erreichen, dem Richter vorzuführen war. Beim Verdacht einer Straftat, die gemeinschaftlich begangen wurde, durfte die Zeit bis zur Vorführung vor den Richter 15 Tage dauern. Während die Verfassung die Mindestzahl der Teilnehmer offenläßt, verlangt die Strafprozeßordnung hier eine Tat, bei der mindestens drei Personen beteiligt sind (Art. 128 II). ${ }^{26}$ Diese langen Fristen, die insbesondere bei der Polizeihaft wegen der Gefahr von Folter gefürchtet waren, waren in den einfachen Gesetzen bereits seit 1992 schrittweise abgebaut worden. Zur Zeit der Verfassungsänderung vom Oktober 2001 lagen sie für Einzeltäter bei 24 Stunden, nur bei Verdacht einer Straftat, die in die Zuständigkeit der Staatssicherheitsgerichte fiel, noch bei 48 Stunden. Bei Straftaten, an denen mindestens drei Personen beteiligt waren, dauerte sie vier Tage mit schriftlicher Genehmigung des Staatsanwalts, konnte aber auf Antrag des Staatsanwalts durch Entscheidung des Richters auf 7 Tage verlängert werden. Bei Verdacht von Taten, die unter die Staatssicherheitsgerichtsbarkeit fielen, konnte in den damals noch vier Notstandsprovinzen durch richterlichen Beschluß eine Verlängerung auf 10 Tage erfolgen. Die Verfassungsänderung vom Oktober 2001 begrenzte jetzt die Haftdauer auf vier Tage, beließ es aber wie vorher bei der Möglichkeit, im Notstandsfall, bei Ausnahmezustand oder Krieg die Dauer zu verlängern (Art. $19 \mathrm{~V}$ ). Die neue verfassungsrechtliche Regelung hatte dann zu Folge, daß im Februar dieses Jahres in der StPO Verlängerungsmöglichkeiten über vier Tage hinaus entfielen und im Staatssicherheitsgerichtsgesetz reduziert wurden (Art. 128 II StPO, Art. 16 II Gesetz Nr. 2845 v. 16.6.1983 - Staatssicherheitsgerichtsgesetz): Nur in den Notstandsprovinzen ist jetzt noch bei Verdacht eines Delikts, das in die Zuständigkeit der Staatssicherheitsgerichte fällt, eine verfassungsmäßig gedeckte Verlängerung auf bis zu sieben Tage möglich (Art. 16 III Gesetz Nr. 2845 v. 16.6.1983). ${ }^{27}$ Notstandsprovinzen sind derzeit jedoch nur noch zwei von 81 Provinzen, nämlich Diyarbakır und Şırnak ${ }^{28}$ und diese voraussichtlich auch nur noch wenige Monate.

Wichtig ist auch eine Verfassungsänderung, die bei einer polizeilichen Festnahme oder einer Verhaftung eine unverzügliche Benachrichtigung von Angehörigen ohne Wenn und Aber garantiert (Art. 19 VI). In der vorhergehenden Fassung gab es noch den Vorbehalt, daß einer Bekanntgabe wegen des Umfangs und Gegenstands der Ermittlungen zwingende Bedenken entgegenstehen könnten. Die sofortige Information von Angehörigen hat aber ebenfalls für einen Festgenommenen eine nicht zu unterschätzende Schutzfunktion, denn Angehörige können sich u.a. um einen Anwalt bemühen. Die Benachrichtigung von Ange-

Dabei kommt es nicht darauf an, daß mindestens drei verdächtige Personen festgenommen werden, sondern daß der Verdacht besteht, daß an der Straftat als solcher insgesamt mehr als drei Personen beteiligt sind. Das ist auch der Grund dafür, daß bei PKK-bezogenen Straftaten i.d.R. die Frist für Gruppen galt.

27

Art. 5, 7 Gesetz Nr. 4744 v. 6.2.2002.

28

In den Provinzen Tunceli und Hakkâri ist der Notstand am 30.7.2002 aufgehoben worden. 
hörigen nach einer Verhaftung war zwar bereits gesetzlich geregelt, mit demselben Vorbehalt, den bisher die Verfassung enthielt; die Benachrichtigung von Angehörigen nach einer polizeilichen Festnahme war aber bisher nur in einer Verordnung vorgesehen. ${ }^{29}$ Für beide Fälle wurde die vorbehaltlose Benachrichtigungspflicht im Februar 2001 durch das Gesetz Nr. 4744 v. 6. 2. 2002 in die StPO aufgenommen (Art. 107, 128 III).

Die neue Verfassungsregelung hat auch den Schutz der Privatsphäre an die Regelungen der EMRK angepaßt und damit verstärkt. Durchsuchungen einer Person, ihrer privaten Papiere (Art. 20) oder ihrer Räume (Art. 21), sowie Beschlagnahmen nach Durchsuchungen waren zwar laut Verfassung an einen gesetzmäßig ergangenen richterlichen Beschluß bzw. in Eilfällen den Beschluß einer gesetzlich zuständigen Behörde gebunden; die einzelnen Ausgestaltungen des Gesetzes waren aber verfassungsmäßig nicht vorgegeben. Gleiches galt auch für Kommunikationsfreiheit und Briefgeheimnis (Art. 22). Die Neuregelung macht hier genaue Vorgaben, bei denen das Vorbild von Art. 8 EMRK deutlich wird: Die verfahrensgemäß ergangene Entscheidung über eine Durchsuchung oder Beschlagnahme darf nur "aus Gründen der nationalen Sicherheit, öffentlichen Ordnung, zur Vereitelung einer Straftat, zum Schutz der öffentlichen Gesundheit oder öffentlichen Moral oder zum Schutze der Rechte und Freiheiten eines anderen" erfolgt sein (Art. 20 II, 21, 22 II). Bei der Eilzuständigkeit anderer Behörden, sprich der Staatsanwaltschaft, wird eine kurze Frist von 24 Stunden für die Vorlage beim Richter gesetzt. Dieser hat binnen 48 Stunden nach Ergehen der Maßnahme seine Entscheidung über ihre Bestätigung oder Aufhebung zu fällen. Eine Nichteinhaltung dieser Fristen hat das automatische Außerkrafttreten eines Beschlusses der eilzuständigen Behörde zur Folge. Die Strafprozeßordnung sieht bei einer Beschlagnahme durch die Staatsanwaltschaft noch eine Frist von bis zu drei Tagen für die Vorlage beim Richter vor (Art. 90 V StPO), bei Postsendungen muß der Richter binnen drei Tagen seit der Beschlagnahme seine Entscheidung treffen (Art. 92 IV StPO). Diese Vorschriften stehen also nicht mehr im Einklang mit der Verfassung und sind zu ändern. Dagegen sieht das vor wenigen Jahren erlassene Gesetz zur Bekämpfung der organisierten Kriminalität (Gesetz Nr. 4422 v. 30.7.1999) in Fällen, in denen wegen Gefahr im Verzug die Staatsanwaltschaft ihrerseits Abhörmaßnahmen oder optische Überwachungsmaßnahmen ohne richterliche Gestattung angeordnet hat, bereits eine Frist von 24 Stunden für die nachträglich einzuholende richterliche Genehmigung vor (Art. 2 V). Damit geht sie also über die verfassungsrechtliche Vorgabe hinaus, die 24 Stunden für die Vorlage ansetzt.

Eine ähnlich deutliche Anlehnung an die EMRK, hier Art. 11, findet sich bei der Neufassung der Artikel zum Vereinsrecht (Art. 33), zur Bildung von Gewerkschaften und Arbeitgebervereinigungen (Art. 51) und zum Versammlungs- und Demonstrationsrecht (Art. 34). Während bisher die grundsätzliche Garantie der Freiheit und eine Reihe technischer Rege- 
lungen im Ernstfall ein Verbot nicht verhinderte, wird jetzt genau festgelegt, aus welchen Gründen Verbote verhängt werden können, nämlich "aus Gründen der nationalen Sicherheit, öffentlichen Ordnung, zur Vorbeugung vor Straftaten, zum Schutze der allgemeinen Moral und allgemeinen Gesundheit sowie zum Schutze der Rechte und Freiheiten anderer". Einschränkungen, die bisher durch Gesetz für die Mitgliedschaft von Beamten in Vereinen vorgesehen waren, werden reduziert; sie dürfen künftig nur noch "in dem Maße, das ihre Aufgaben erfordert" bestehen (Art. 33 VI).

Viel beachtet wurde die Streichung des sogenannten Sprachenverbots aus den Artikeln über die Freiheit der Äußerung und Verbreitung einer Meinung (Art. 26) sowie der Pressefreiheit (Art. 28). Hier hieß es, Veröffentlichung bzw. Verbreitung von Meinungen in einer durch Gesetz verbotenen Sprache sei unzulässig. 1983 war ein Gesetz erlassen worden, das Publikationen und Demonstrationen in einer Sprache verbot, die nicht erste Staatssprache eines von der Türkei anerkannten Landes war. Diese gewundene Formulierung zielte auf das Kurdische. Es verbot keineswegs, wie häufig in Deutschland angenommen, die Benutzung des Kurdischen im privaten Umgang. Das wäre bei einer kurdischen Bevölkerung von ca. 12 Millionen gar nicht vorstellbar gewesen. Es betraf vielmehr den Gebrauch des Kurdischen in Publikationen und im öffentlichen Bereich der Meinungsverbreitung. Strafrechtliche Verurteilungen nach diesem Gesetz gab es fast keine. Das Gesetz wurde 1991 aufgehoben, und damit schienen prima facie auch die entsprechenden Verfassungsbestimmungen leer zu laufen. Im Istanbuler Bücherbasar fand man nach Aufhebung des Verbots weit weniger kurdische Publikationen als vorher. Es sind wohl Käufer weggeblieben, die vorher aus bewußter Opposition kurdische Bücher gekauft hatten. Rein wirtschaftlich tragen sich kurdische Publikationen meist nicht, der Leserkreis ist zu klein. Trotzdem stand das Problem der Verwendung des Kurdischen weiter auf der Tagesordnung, nur unter einem anderen Gesichtswinkel. Die Verfassung stellt wie bisher in Art. 42 VIII fest, daß in den Schulen keine andere Sprache als türkisch als Muttersprache gelehrt werden dürfe. Die Verwendung der kurdischen Sprache bzw. der Wunsch, kurdisch zu lernen oder gar kurdischen Schulunterricht zu ermöglichen, wurde eben nicht isoliert als gewissermaßen privater Wunsch angesehen, diese Sprache aus welchen Gründen auch immer zu pflegen, sondern rasch als gezielte Unterstützung separatistischer Bestrebungen, als Kampfmittel der PKK oder mit ihrem neuen Namen, der KADEK. Zu Beginn des Jahres 2002 kam es gehäuft zu Demonstrationen und Einreichungen von Gesuchen mit dem Anliegen, Unterricht in kurdischer Sprache einzurichten. Die Strafverfolgungsbehörden werteten das als eine Kampagne der PKK und leiteten zahlreiche Ermittlungsverfahren wegen Unterstützung einer bewaffneten Bande (Art. 169 StGB) ein. Auch hier bereitet das Gesetz Nr. 4777 v. 3. 8. 2002 den Weg für die von der EU schon lange angemahnte Öffnung (Art. 11). Es änderte das Gesetz über die Lehre und Erziehung in Fremdsprachen (Gesetz Nr. 2923 v. 14.10.1983) und schuf die Möglichkeit, private Kurse für das Erlernen von Sprachen und Dialekten einzurichten, die traditionellerweise von türkischen Staatsbürgern im Alltag gesprochen werden. Dabei sollen sie den Vorschriften des Privatschulgesetzes (Gesetz Nr. 625 v. 8.6.1965) 
unterliegen. Das Nähere soll eine Verordnung regeln, die binnen eines Jahres zu ergehen hat. Jedoch erklärt das Gesetz ausdrücklich, dass derartige Kurse nicht gegen die in der Verfassung festgelegten Grundlagen der Republik und gegen die unteilbare Einheit von Staatsgebiet und Staatsvolk verstoßen dürfen. Diese Bestimmung läßt immer noch das Mißtrauen erkennen, das staatlicherseits gegen die Förderung der kurdischen Sprache besteht. Leicht wird schon in der Tatsache der Verwendung des Kurdischen als solcher eine ideologische Festlegung auf separatistische Ziele gesehen. ${ }^{30}$ Die eingeleiteten Strafverfahren wegen des Wunsches nach Kurdischunterricht sind jedoch nach diesen Gesetzesänderungen zu beenden.

Eine ähnliche Änderung erfolgte in Art. 4 I des Gesetzes über die Errichtung und Sendungen von Rundfunk- und Fernsehanstalten (Gesetz Nr. 3984 v. 13.4.1994). Noch im Mai 2002 war eine Änderung des Art. 4 erfolgt (Gesetz Nr. 4756 v. 15.5.2002), die die Bedeutung der türkischen Sprache in den Vordergrund gerückt hatte und Sendungen, sogar Musiksendungen, nur in fremden Sprachen zuließ, die einen Beitrag zur universellen Wissenschaft und Kultur geleistet hatten - was Sprachen wie Kurdisch ausschloß. Weniger als drei Monate später schuf das Gesetz die Möglichkeit, wie bei den Sprachkursen auch Rundfunk- und Fernsehsendungen in Sprachen und Dialekten, die im Alltag traditionell von türkischen Staatsbürgern gesprochen werden. ${ }^{31}$ Auch hier findet sich allerdings dieselbe "Staatstreueklausel" wie beim Sprachunterricht. Aber hinter allen politischen Erwägungen ist dabei etwas anderes zu bedenken: die technologische Revolution der letzten Jahre. In Ländern wie der Türkei waren schon lange Radio und Fernsehen weit wichtiger als Zeitungen oder gar Bücher. Mittlerweile gibt es unzählige private Fernseh- und Rundfunkanstalten, die auch grenzüberschreitend senden können und es tun, man denke nur an den bekannten PKK-nahen Sender Medya TV. Dazu kommt in den letzten Jahren der Siegeszug des Internet. Die technische Entwicklung ist hier über die Auffassungen und Wünsche staatlicher Stellen hinweggegangen. Ein Verbot der kurdischen oder einer anderen Sprache ist schon aus diesen Gründen heute ein Anachronismus.

Erschwert wurde auch die Möglichkeit eines Parteienverbots. Dazu muß man vorwegschicken, daß in der Türkei Parteien von vornherein nicht so stabile und etablierte Gebilde sind wie in Deutschland. Laufend werden Parteien gegründet und zerfallen, häufig wechseln Parlamentsabgeordnete die Partei. Auch ein Parteienverbot hat in der Türkei bei weitem nicht das Gewicht, das es etwa in Deutschland hätte. Bis auf einige wenige Führungspersönlichkeiten behalten auch die Abgeordneten verbotener Parteien ihr Abgeordnetenmandat. Das Vermögen der Partei wird regelmäßig schon während eines laufenden Verbotsverfahrens so "verteilt", daß bei einer tatsächlichen Schließung nicht mehr viel

30

Da Texte in kurdischer Sprache den Strafverfolgungsbehörden meist sprachlich nicht zugänglich sind, sind Verfahren als Folge der Auswertung seines Inhalts dagegen selten.

Gesetz Nr. 4777 v. 3.8.2002. 
davon erreichbar ist. Nachfolgegründungen sind an der Tagesordnung, und böse Zungen behaupten, nach einem Parteienverbot würde nur das Messingschild an der Tür des Parteibüros ausgetauscht. - Nach wie vor dürfen Parteien nicht gegen die Grundsätze der Unabhängigkeit, der unteilbaren Einheit von Staatsvolk und Staatsgebiet, die Menschenrechte, die Prinzipien von Gleichheit und Rechtsstaat, die nationale Souveränität und die Prinzipien der demokratischen und laizistischen Republik verstoßen, keine Diktatur anstreben oder verteidigen und nicht zur Begehung von Straftaten auffordern. Eine Schließung der Partei erfolgt durch Beschluß des Verfassungsgerichts, wenn derartige Verstöße zu einem Brennpunkt der Aktivitäten werden (Art. 69). Neu ist, daß die Verfassung festlegt, was unter einem Brennpunkt von Aktivitäten zu verstehen ist. "Eine politische Partei wird zum Brennpunkt solcher Aktivitäten, wenn entsprechende Taten von Parteimitgliedern in großem Umfang begangen werden und dies vom Großen Kongreß, dem Vorsitzenden, von Entscheidungs- und Verwaltungsorganen der Parteizentrale, der Hauptversammlung oder der Führung der Parlamentsfraktion stillschweigend oder ausdrücklich gebilligt wird oder solche Taten von den genannten Parteiorganen selbst bewußt und gewollt begangen werden." Von dieser genauen Definition erwartet man sich eine Einschränkung etwaiger Parteiverbote. Als mildere Maßnahme gegenüber einem Parteienverbot wird die Möglichkeit geschaffen, bei einer erheblichen Zahl von Verstößen der Partei staatliche Finanzierungen ganz oder teilweise zu entziehen (Art. 69 VII). ${ }^{32}$

Durch eher technisch erscheinende Änderungen werden die Möglichkeiten verstärkt, parlamentarische Ermittlungsverfahren wegen etwaiger strafrechtlicher Verfehlungen gegen den Ministerpräsidenten oder Minister einzuleiten, die in der Vergangenheit - man denke nur an Tansu Çiller - immer wieder unterdrückt worden waren. Über das Verlangen auf Einleitung eines solchen Verfahrens hat das Parlament nunmehr in geheimer Abstimmung zu entscheiden, was die Abgeordneten möglichem Druck entzieht (Art. 100 I). Der Bericht über das Ermittlungsverfahren, das bei einem positiven Beschluß von einem Gremium von 15 Abgeordneten durchzuführen ist, ist nach zwei, spätestens nach vier Monaten dem Präsidium des Parlaments vorzulegen (Art. 100 II). Dieses wird für das weitere Vorgehen an Fristen gebunden: Binnen 10 Tagen ist der Bericht an die Abgeordneten zu verteilen, binnen 10 weiteren Tagen darüber zu debattieren und geheim darüber abzustimmen, und bei positivem Ergebnis der Betroffene an das Verfassungsgericht in seiner Eigenschaft als Staatsgerichtshof $^{33}$ zu überstellen. (Art. 100 III). Der Versuchung, durch eine Verschleppung des Verfahrens die Angelegenheit in Vergessenheit geraten zu lassen, soll so ein Riegel vorgeschoben werden.

Entsprechende Änderungen im Parteiengesetz sind mittlerweile durch Art. 4 Gesetz Nr. 4748 v. 26.3.2002 erfolgt. sonstiger im Gesetz genannter Spitzenämter im Zusammenhang mit ihrem Amt begangen haben, Art. 148 III. 
Bemerkenswert ist die Aufhebung des Übergangsartikels 15, der die Geltendmachung der Verfassungswidrigkeit von Gesetzen verbot, die in der Zeit der Militärregierung (12.9.1980 - 6.12.1983) ergangen waren. Das könnte dazu führen, daß es in der nächsten Zeit zu einer Reihe diesbezüglicher Verfahren kommen wird. Eine große Zahl von Entscheidungen des türkischen Verfassungsgerichts zeigt, daß dieses keine Scheu kennt, gegenüber Regierung und Parlament die in der Verfassung festgeschriebene Rechtsstaatlichkeit einzufordern. Und Entscheidungen oder auch nur absehbare Entscheidungen des Verfassungsgerichts haben den türkischen Gesetzgeber immer wieder zum Handeln veranlaßt. Gewiß sind einige der Gesetze aus der Zeit der Militärregierung schon einschneidend geändert worden, trotzdem gibt die Aufhebung des Übergangsart. 15 die Chance zu weiteren Reformen.

Der Nationale Sicherheitsrat, der weithin als das eigentliche Zentrum der Macht angesehen wird, bestand bisher aus dem Staatspräsidenten, dem Ministerpräsidenten, den Ministern für Verteidigung, Inneres und Äußeres, dann aber aus dem Generalstabschef, den drei Kommandeuren der Land-, See- und Luftstreitkräfte. Die militärischen Mitglieder hatten also ein starkes Gewicht. Mit der Absicht, ihren politischen Einfluß zurückzudrängen, wurde der Nationale Sicherheitsrat um fünf weitere zivile Mitglieder, nämlich die stellvertretenden Ministerpräsidenten und den Justizminister, vermehrt und seine Beschlüsse von bindenden Vorgaben zu Empfehlungen herabgestuft (Art. 118). Ob das im Ergebnis tatsächlich an den Realitäten etwas ändert, bleibt abzuwarten.

Im Bereich der Änderungen, die das gesamtgesellschaftliche Gefüge angehen, ist die Gleichstellung von Mann und Frau die wichtigste, die interessanterweise aber nicht beim Gleichheitssatz, sondern in einem Artikel über den Schutz der Familie (Art. 41) angesiedelt ist. Wenige Wochen danach trat bereits ein neues Zivilgesetzbuch in Kraft (Gesetz Nr. 4721 v. 22.11.2001), bei dem die Durchsetzung der Gleichheit von Mann und Frau auch im einfachen Gesetz einer der Schwerpunkte ist. Die Gleichheitsgrundsatz tritt jetzt noch in einem weiteren Verfassungsartikel in Erscheinung: künftig erhält auch das Kind einer türkischen Mutter und eines ausländischen Vaters automatisch die türkische Staatsangehörigkeit (Art. 66 II).

Zu den Verfassungsartikeln, deren Änderung keine Mehrheit fand, gehört Art. 90. Dieser sieht zwar bisher schon vor, daß völkerrechtliche Verträge, die verfahrensgemäß in Kraft gesetzt worden sind, in der Türkei Gesetzeskraft haben und vor dem Verfassungsgericht nicht als verfassungswidrig angegriffen werden können (Art. $90 \mathrm{VI}$ ). Das gilt z.B. für die EMRK. Die Vorschrift legt aber nicht fest, welchen Rang in der Normenhierarchie ein solcher, ins türkische Recht übernommener Vertrag haben sollte. Welches Recht gilt nun, wenn eine Norm des nationalen Rechts eine andere Regelung vorsieht? Die geplante Änderung sollte nun im Falle eines solchen Widerspruchs dem völkerrechtlichen Vertrag Vorrang vor dem nationalen Recht einräumen. Dieser Vorschlag wurde aber bereits in erster Lesung abgelehnt. 
Ein weiterer abgelehnter Änderungsvorschlag betraf die Voraussetzungen für eine Kandidatur zum Parlament. Die Diskussion darüber rief in der Türkei deshalb besonderes Interesse hervor, weil sie sich anschaulich an dem sehr populären ehemaligen islamistischen Oberbürgermeister von Istanbul, Tayyip Erdoğan, festmachte. Erdoğan hatte vor einigen Jahren in einer Rede ein Gedicht von Ziya Gökalp zitiert und war deswegen gem. Art. 312 II wegen Aufhetzung einer Bevölkerungsgruppe gegen eine andere unter Hinweis auf Unterschiede in der Religion zu einer Haftstrafe verurteilt worden. Da laut Art. 76 der Verfassung eine Verurteilung wegen Teilnahme an ideologischen und anarchistischen Taten sowie die Anstiftung und Aufhetzung dazu eine Wahl zum Abgeordneten für immer ausschließt und Art. 312 eine derartige Tat darstellt, kann Erdoğan sich nicht als Kandidat bei Parlamentswahlen aufstellen lassen. Diese Beschränkung war lange heiß diskutiert. Der Entwurf der Verfassungsänderung wollte in dem Artikel 76 die Formel von der Verurteilung wegen Teilnahme an ideologischen und anarchistischen Taten sowie die Anstiftung und Aufhetzung zu solchen Taten durch die Formel "terroristische Taten" ersetzen. Damit wäre bei einer Verurteilung gem. Art. 312 StGB, die keine terroristische Tat darstellt, der Weg zu einer Kandidatur für Erdoğan und generell auch für andere wegen Meinungsäußerungsdelikten verurteilte Kandidaten frei gewesen. Obwohl es zunächst so aussah, daß möglicherweise die notwendige Mehrheit der Abgeordneten für diese Änderung zu gewinnen sei, wurde in der geheimen Abstimmung die erforderliche Stimmenzahl nicht erreicht. Auch eine weitere diskutierte Änderung kam nicht durch: Mitglieder einer Partei, deren Äußerungen oder Aktivitäten Ursache für deren Verbot waren, dürfen nach Veröffentlichung des entsprechenden Urteils des Verfassungsgerichts fünf Jahre lang nicht Gründer, Mitglied oder gar Mitglied des Vorstands oder des Aufsichtsorgans einer Partei werden (Art. 69 III). Auch eine Verkürzung oder gar Aufhebung dieses "Politikverbots", bei dem der wohl prominenteste Betroffene der ehemalige islamistische Ministerpräsident Erbakan ist, fand keine Mehrheit.

Der türkische Gesetzgeber ist in der letzten Zeit sehr aktiv gewesen. Weitere große Vorhaben, z.B. eine neue Strafprozessordnung und ein neues Strafgesetzbuch, sind geplant. Die Änderung der Verfassung ist also eingebettet in eine ganze Reihe von Änderungsgesetzen, wenn sie auch besondere Aufmerksamkeit erregte. Sie ist ein Schritt auf dem Weg in die EU, aber der Weg ist noch lang, und viele Veränderungen in Staat und Gesellschaft werden noch folgen müssen. 